\title{
Assessment on Utilization of Health Management Information System at Public Health Centers Addis Ababa City Administrative, Ethiopia
}

\author{
Tsedale Adane, Trhas Tadesse, Getabalew Endazenaw \\ Yekatit Hospital Medical Colleges, Addis Ababa, Ethiopia \\ Email address: \\ tsdaladne@gmail.com (T. Adane), ttrhas@gmail.com (T. Tadesse), eyobgetabalew@gmail.com (G. Endazenew) \\ To cite this article: \\ Tsedale Adane, Trhas Tadesse, Getabalew Endazenaw. Assessment on Utilization of Health Management Information System at Public \\ Health Centers Addis Ababa City Administrative, Ethiopia. Internet of Things and Cloud Computing. Vol. 5, No. 1, 2017, pp. 7-18. \\ doi: 10.11648/j.iotcc.20170501.12
}

Received: December 16, 2016; Accepted: March 1, 2017; Published: March 18, 2017

\begin{abstract}
Health Management Information System (HMIS) has sub system under HIS is specially designed to assist the management and planning of health programmes opposed to delivery of care that provides decentralized decision making and planning. However, decentralized informational management is at infancy stage in Ethiopia. Hence, to ensure the effective and efficient utilization of a system, periodic assessment becomes imperative at this juncture. The study aims in assessing utilization of HMIS at Public Health Centers in Addis Ababa. Institution based cross sectional quantitative and qualitative study was conducted from July to September 2011. The sample size was calculated using single population proportion formula and a total of 416 health professionals were included. Frequencies, proportion and summary statistics were used to describe the study population in relation to relevant variables. Bivariate and multivariate analyses were also be carried out to see the effect of each independent variable on the dependent variable. 416 respondents respond to the questionnaire with $98 \%$ response rate. The current utilization rate of HMIS at health centers were about $41.7 \%$. The total outcomes of the generated routine data were compiled using tally sheet, registers, and reports, and in combination of these formats. The combined utilization of the mentioned formats constituted 199 (48.8\%). The odds ratio result indicated that the provisions of technical supports, presence of computers, generated data at department level and practice of conversion of data into information enhanced the utilization of HMIS (AOR=1.69, 95\%CI:1.09-2.63), (AOR=2.08, 95\%CI:1.29-3.35) $(\mathrm{AOR}=2.78,95 \% \mathrm{CI}: 1.65-4.68)$, and $(\mathrm{AOR}=3.44$, 95\% CI:(1.82-6.51) respectively.
\end{abstract}

Keywords: Utilization, HMIS, Public Health Facility

\section{Introduction}

Health management information system (HMIS) is a process in which health data are recorded, stored and processed for policy-making, planning, implementation and evaluation of health programs for appropriate use of resources at all levels of the health system [1].

As WHO has been advocating for the aforementioned idea and clearly stated it, information is one of the fundamental components for better and effective health service delivery at all management levels of health services. Furthermore, effective health management is also a prerequisite for productive health services.

In addition developing quality health information management system is crucial to 1 render fast and efficient service to patients/clients. Good information management is also a prerequisite for increasing the effectiveness of health services. WHO has identified health information system (HIS) as critical to achieve the campaign health for all by the year 2000 \& clearly links it to improve HIS [2].

Developing countries are reported to have a large amount of unreliable health data, poor human resources, and poor information technology infrastructure [5] and defective Health Management Information Systems [6]. Therefore, it becomes imperative to improve the processes of data handling in order to extract useful information for health 
planning, decision making, and resource allocation.

The Ethiopian National Health Information System assessment report of 2007 identified that HMIS is among the major problems of the sector. It is characterized by burdensome and small qualified staff, poor and fragmented information flow and with no integration among the various subsystems. This resulted in conflicting reports and poor quality of data in terms of accuracy and timelines, preventing information users from effective utilization of information for decision making [7].

The management of health information has been in the process of revitalization in the past five years in Addis Ababa, public hospital and health center. Despite this activity standardization, customization and computerized information system of the public health institutions are not so far satisfactory [6]. The principal objective of the FMOH in its strategic plan were to define the minimum standard of inputs required for HMIS at different levels of the health system and to initiate and sustain regular program review and feedback system.

Therefore, how far these objectives were achieved must be investigated to activate and intervene in the health information system should be done. Although few studies have been conducted on the utilization of HMIS in Public Hospitals Addis Ababa [8] but there was no pertinent study conducted on HMIS at health centers in Addis Ababa as far as my literature review is concerned. This requires imperative survey and analysis of the utilization of HMIS. Therefore this paper will try to investigate the utilization of the institutionalized reformed HMIS in public health centers under Addis Ababa city Administrative health bureau.

Health Management Information System is one of the seven major components of Ethiopia's Health Sector Development Program III since 2005/2006 (1998E. C) [24]. This indicates that issue of health management information system is receiving due attention given by the Ethiopian government. However, the achievements so far are not satisfactory.

The reason for this could be listed as follows the considerable problems of incompleteness, untimely, redundancies of records, diversified format to collect data, and inconsistency of facts are some of the challenges in the health information system in Ethiopia [25].

HMIS is the early step in the strategy for strengthening and improving it. This investigation contributes to the improvement of current HMIS at Health facilities in A. A. Therefore to ensure the effective and efficient utilization of a system, periodic assessment is very important at this juncture.

\section{Objective}

\subsection{General Objective}

To assess health management information system utilization in all public health centers in Addis Ababa.

\subsection{Specific Objectives}

To describe Routine Data Generation

To Determine the Use of Information

To Identify Factors that Affects Utilization of Health Management Information System

\section{Methods and Materials}

The study was conducted in Addis Ababa City Administrative (A. A. C. A.) Public health centers. Addis Ababa, capital city of Ethiopia, situated at the heartland of Ethiopia, in an area of 540 square kilometers, with latitude $9^{0}$ North and longitude $38^{\circ}$ East, A. A is divided in to 10 administrative sub cities and 99 Kebeles. Addis Ababa is one of the two city administration of the Federal Democratic Republic of Ethiopia. Addis Ababa Health Bureau is responsible for the overall Health activity in the city. The City has 13 Public hospitals (5 under the bureau) and 26 Health Centers. In addition there are 15 Private General, 07 specialized Hospitals as well as 89 Higher, 110 medium, 98 lower, 90 specialized clinics (ear, nose, throat (ENT), ophthalmology, Dentistry) etc (26). The 26 health centers are found less than 10 sub city in Addis Ababa. Addis Ababa has a total population 2,738,248 of whom $1,304,518$ (47.6\%) were men and 1,433,730 (52.4\%) women (27) Potential health service coverage of A. A is $86.45 \%$ (28). Institution based cross-sectional study design using both quantitative and qualitative approach was employed. Further observation was don using check list to support the result of quantitative methods from July to September 2011. The source population for the study was all health workers who have been working in the 26 public health centers in Addis Ababa city administrative. Selected health professionals who served at least for six months were included and who are ill, during data collection period were excluded. Sample size was determined using single population proportion formula for cross-sectional study. Taking 52\%, a value obtained from a related research which was conducted on Assessment of health management information system at Public Hospital in Addis Ababa [8]. To obtain maximum sample size at $95 \%$ certainty and a maximum discrepancy of $\pm 5 \%$ between the sample and the population. Adding 5\% non-response rate, sample size became 416. Regarding the sampling procedure, random sampling was used to select study subjects using computer generated random number by taking the list of health professionals from all public health centers human resource administration department as a sampling frame. If the selected health professional is not available for three times repeated trial during data collection period, the next person in the frame was selected. Utilization of HMIS at public Health Centers was the dependent variable and Socio demographic characteristics (sex, age, and educational level, and salary, year of service, occupation), Resource for HMIS, Data completion process and People who collect and use the data (attitudes, and motivation) were independent 
variable.

Self administered structured questionnaire was used to collect quantitative data as well as checklist for observation was used to identify data generation protocols and, the availability of HMIS resources. The questionnaire was prepared in English and translated to Amharic then back to English to assure consistency. Three days training was given for three supervisors (two heath officers and one BSc Nurses) and five Nurses data collectors (BSc and Diploma) to facilitate data collection process. Pretest was conducted at "'Debrezeite" health center andnecessary adjustments were done prior to the actual study time.

The collected data were entered, after being encoded using EPI info version 3.5.1 and finally analyzed using SPSS version 16. Data cleaning was performed by generating frequencies table to check, accuracy, consistencies, missed values and variables. Errors identified during data entry were corrected after revision of the original completed questionnaire. Descriptive statistics was used to explain the study population in relation to relevant variables. Associations between dependent and independent variables were assessed and their strength was presented using odds ratios and 95\% confidence intervals. Both bivariateand multivariate logistic regression was used to assess the association between outcome and explanatory variables. Variables having $\mathrm{p}$ value up to 0.2 (as cut off point) in the bivariate analysis were fitted in to the multi variate model. The results were presented using texts, tables, charts, and figures. For qualitative data (observational findings), thematic analyses with a framework approach were employed. Thematic analysis has flexible in nature which is useful to organize \& describe data in detail searching themes across the data to find out repaired patterns. It is an approach to deal with data that involves the creation $\&$ application of codes to data.

The study was carried out after getting approval from the ethical clearance committee of University of Gondar. Data collection was preceded after securing written consent from Addis Ababa health bureau, through formal permission letter obtained from the University of Gondar. After the purpose and objective of the study had been elaborated to each informant, verbal consent was obtained from each study participants. Participants were also being informed that participation was on voluntary basis and they were aware of their ethical rights that they can stop or leave from the participation at any time if they felt any inconvenience or discomfort. In order to keep confidentiality of any information provided by study subjects, the data collection procedure was anonymous.

Operational Definition

Utilization of health Information system: were assessed by matrixes which include: using information for decision making to take immediate action, feedback from respective supervisors, calculation of area coverage and preparation of maps, presence of key indicators with charts or tables (indicators were not expected to be same that is it varies from one unit to the other unit and Presentation of achievements of targets at the health facilities.

So, the study units will be considered as utilizing health information system when they are practicing at least three of them out of the five criteria's listed above. (23)

Standard MRU:Area for health center room's specified $\geq$ $24 \mathrm{~m}^{2}$; for hospitals $\geq 60 \mathrm{~m}^{2}$

Standard shelves Specification of standard rooms with height of 3 meter.

Shelves: height, length, width, columns, rows and cells

1. Height $2.75 \mathrm{~m}$, length $2 \mathrm{~m}$

2. Columns 4 , rows $8-10$

3. Cell: ht $25 \mathrm{~cm}$, width $35 \mathrm{~cm}$, length $50 \mathrm{~cm}$, 'based on size of new folder and preferred size of a shelve'

Shelf specification in rooms with smaller height is similar, except for its height, the height can vary, but there should be $25 \mathrm{~cm}$ space between the upper age (top) of a shelve and roof of a room, as mentioned earlier.

MPI boxes: In a hospital: overall ht $1.5 \mathrm{~m}$, length 2 meter width, with at least 26 pockets for alphabet, but in practice some alphabets repeat more than the others do therefore 40 boxes (pockets) are advisable. In a health center, the only difference is overall length; $1 \mathrm{~m}$ and pockets (boxes) 32 (adapted from Report paper fact sheet of FMOH).

Paramedics:A professional who works in a health field in an auxiliary capacity to a physician as by giving injections and taking X-rays (taken from free Merriam-Webster Dictionary).

\section{Result}

Socio-demographic characteristics of the respondents

26 public health centers found in Addis Ababa city were included in the study. A total of 416 respondents were included making the overall response rate of $98 \%$. The sex distribution of individuals working in the study units showed that $225(55.1 \%)$ were female. Among the total respondent $306(75 \%)$ were within the age of $22-32$ years old with a mean age of 30 years, $117(28.1 \%)$ of them have a salary ranging from $1233-2250 \mathrm{ETB}$ and $275(67.5 \%)$ of them also have 1-5 year of services.

With regard to level of education, health workers with degree and above constituted 192(47\%) and diploma and below were $216(53 \%)$. The majorities of the respondents were diploma holders in different health professionals and the least number had Medical Doctors (1(.2\%)). Health professionals involved in the study had nurse diploma (155 (38\%)), Bsc Nurses (95 (23.3)), Health Officers (55(13.5)), paramedics Diploma (63 $(15.5 \%))$, paramedics degree $(39(9.5 \%))$, andmajority of health professionals were currently working at OPD 91(22.3\%), MCH 70 (17.2\%), and HIV- clinic $41(10 \%)$; the rest working in different department like laboratory, pharmacy, EOPD, EPI, and inpatient.

From the total respondent's position in the organization, majority of the respondent were $273(66.9 \%)$ technical staff followed by $135(33.1 \%$ ) department head (Table 1$)$. 
Table 1. Socio-demographic characteristics of study subjects in the health centers of A. A. July $2011(n=408)$.

\begin{tabular}{lll}
\hline Variables & Frequency & Percent (\%) \\
\hline Sex & 183 & \\
Male & 225 & 44.9 \\
Female & 408 & 55.1 \\
Total & & 100 \\
Age & 93 & \\
$20-24$ & 163 & 22.8 \\
$25-29$ & 65 & 40.1 \\
$30-34$ & 34 & 15.9 \\
$35-39$ & 23 & 8.3 \\
$40-44$ & 17 & 5.6 \\
$45-49$ & 13 & 4.1 \\
$>50$ & 408 & 3.2 \\
Total & & 100 \\
Salary & 100 & \\
$<1249$ & 146 & 24.5 \\
$1250-2249$ & 162 & 35.8 \\
$>2250$ & 408 & 39.7 \\
Total & & 100 \\
Year of service & & \\
$1-5$ & 275 & 67.4 \\
$6-10$ & 75 & 18.4 \\
$11-15$ & 29 & 7.1 \\
$16-20$ & 16 & 3.9 \\
$21^{+}$ & 13 & 3.2 \\
Total & 408 & 100 \\
\hline & &
\end{tabular}

\begin{tabular}{lll}
\hline Level of education & & \\
Bachelor Degree & 192 & 47 \\
Diploma & 214 & 52.5 \\
Certificate & 2 & .5 \\
Total & 408 & 100 \\
Respondents Occupation in the organization & & \\
Medical Doctors & 1 & .2 \\
Diploma Nurses & 155 & 38 \\
BSc Nurses & 95 & 23.3 \\
Health Officers & 55 & 13.5 \\
Paramedics Diploma & 63 & 15.5 \\
Paramedics BSc & 39 & 9.5 \\
Total & 408 & 100 \\
Currently working departments & & \\
OPD & 91 & 22.3 \\
F/P & 26 & 6.4 \\
EOPD & 29 & 7.1 \\
In patients & 8 & 2.0 \\
Laboratory & 36 & 8.8 \\
MCH & 70 & 17.2 \\
Pharmacy & 36 & 8.8 \\
Tb \& Leprosy & 14 & 3.4 \\
Data clerk /statics & 9 & 2.2 \\
EPI & 30 & 7.4 \\
Others & 18 & 4.4 \\
Total & 408 & 100 \\
Respondent position in the organization & & \\
Department Head & 135 & 33.1 \\
Technical Staff & 273 & 66.9 \\
Total & 408 & 100 \\
\hline
\end{tabular}

Data generation process:The routine health data were gathered using by register, report, and tally sheet. The data generation mechanisms in the studied health centers were predominantly based on tally sheet, registers, and reports in combination that accounts $199(48.8 \%)$.
Data collection tools used by studied health facilitites

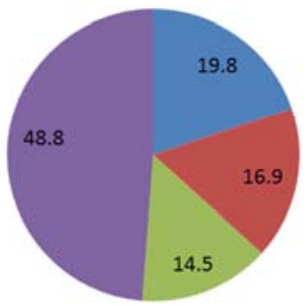

negister

= Report

= Tally sheet

- Register/tally sheet/report

Figure 2. Data collection tools used by studied health facilities, July 2011.

Regarding the convenience of the recording tools to register or collect data, $194(47.5 \%)$ of the respondent reply there was incompleteness and difficulty to understand the formats while $134(32.8 \%)$ replied the formats were simple and completed and $80(19.6 \%)$ were not commented on this issue. For the question whether there were shortages of paper formats to gather health data or not, majority of respondents $273(67 \%)$ replied that there was no shortage of recording tools, but the rest said, sometimes there is shortage of tools within the last three months period.

It has been observed that respondents in the study health facilities replied that $339(83.1 \%)$ of them register the health activities /services rendered routinely and 355(87\%) of the study units have taken data collection as part of their dutyand $339(83.1 \%)$ of the response of the respondents indicated that the workers are committed to collect the health data. The health data were collected almost all by health professionals with themselves $323(79.2 \%)$ other health professionals and data clerk were $118(28.9 \%)$ and 334(81.9\%) of respondents said formats were legibly and completely filled by the health professionals. Majority of health facilities have computer program to enter data, $256(62.7 \%)$ of the respondents in the study replayed. Most of the respondent replied that 261 (64\%) collected data would be analyzed and displayed in graphs and charts while 147 (36\%) of them repliedthat the data was not analyzed and displayed in graphs or charts (Table 2).

Table 2. Registration of health activities and data analysis at health centers A. A, July 2011.

\begin{tabular}{lll}
\hline Variables & \multicolumn{2}{l}{ Frequency Percent (\%) } \\
\hline Register the health activities & & \\
Routinely & 339 & 83.1 \\
Rarely & 28 & 6.8 \\
Not at all & 11 & 2.7 \\
DK (do not know) & 30 & 7.4 \\
Data analyzed and displayed & & \\
Graph & 68 & 16.7 \\
Chart & 62 & 15.2 \\
Both & 131 & 32.1 \\
No analyzed & 147 & 36 \\
\hline
\end{tabular}

In some of the health centers $(32.1 \%)$, the reports were displayed by graph, chart and in both (figure 3 ). 

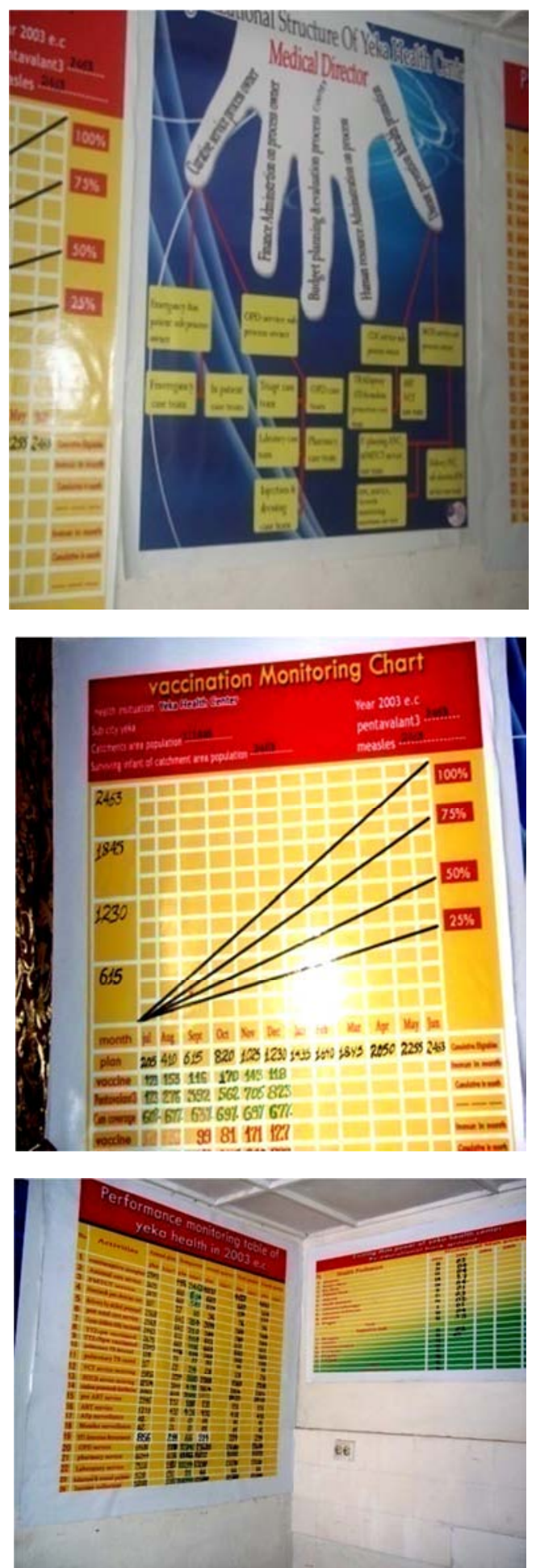

Figure 3. One of the Health Centeruse displayed information at, A. A. July 2011.

The data collected and documented using paper formats and sometimes it may be directly feed into the computer formats. In the studied health facilities, 178(43.6\%) were used only paper formats to generate client health information and $164(40.2 \%)$ of the health centers used both computer formats and paper formats in combination while 66(16.2\%) of them used only computer formats to collect and document the health information of their client (figure 4)

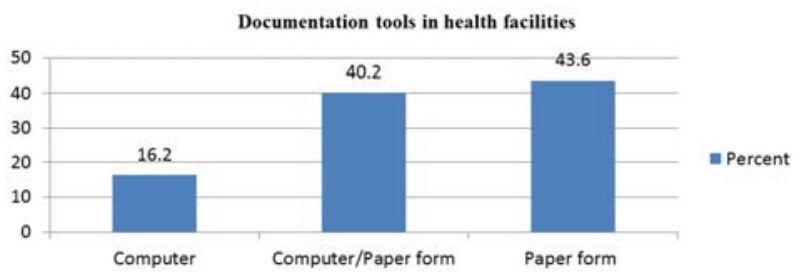

Figure 4. Percentage of documentation forms used by the health centers, July 2011.

Utilization of Information:

Out of the 408 respondents, $170(41.7 \%)$ have met three and more criteria for information use. (figure 5).

\section{Utilization of HMIS}

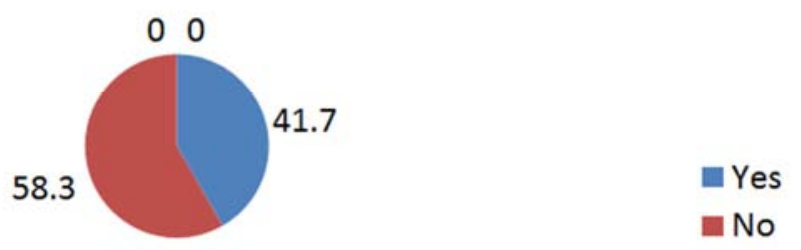

Figure 5. Percentage of HMIS utilization in health centers July 2011.

About a quarter of the respondents 111(27.2\%) reported that they use generated information's for all intended purposes including; to give information to the user, to compare it with the previous, for Monitoring/Evaluation of programs to take immediate action and for planning purposes and $46(11.2 \%)$ of the respondents forward the generated information to upper level. (Table-3)

Table 3. Respondents' use of generated information at their departments, Addis Ababa health centers July 2011.

\begin{tabular}{lll}
\hline Variables & Frequency & Percent (\%) \\
\hline To give information to the user & 103 & 24.9 \\
To compare it with the previous & 76 & 18.5 \\
Monitoring/Evaluation of programs to & 64 & 15.6 \\
take immediate action & & \\
Planning & 85 & 20.7 \\
Decision making & 62 & 15.1 \\
For ward upper level & 46 & 11.2 \\
For all & 111 & 27.2 \\
\hline
\end{tabular}

In addition the generated and analyzed data also used by the health institution itself 253(62\%)and 46(11.3\%) higher managerial bodies and both health center and higher offices 87 (21.3) while 22(5.4\%) of respondents answered they had no idea about its utilization.

Table 4. The user ofgenerated information at health centers A. A July 2011.

\begin{tabular}{llll}
\hline & Yes & No & Total \\
\hline The generated and analyzed data Used by & & & \\
Health institution itself & 99 & 154 & 253 \\
Higher management & 12 & 34 & 46 \\
Both the health center and higher offices & 50 & 37 & 87 \\
Not used by the health center \& other offices & 9 & 13 & 22 \\
Total & 170 & 238 & 408 \\
\hline
\end{tabular}

Regarding the conversion of health data into information $305(74.8 \%)$ respondents replied that they had knowledge 
and practice that the generated data were converted into information wheras $103(25.3 \%)$ of them did not have idea whether the data converted into information or not. On the question at what frequency the health data converted into health information, majority of the respondents 198(63\%) replied that it has been done at monthly basis.

For the question whether there was guidelines for HMIS in health facilities or not $211(51.7 \%)$ of the respondents replied that the health facilities had guide lines and user manuals to run their activities effectively. Yet a significant number of respondents $197(48.3 \%$ ) replied that they did not have idea whether the heath unit had the mentioned materials.

Most of the respondents replied that 323(79.2\%) of the health facilities had clear annual plans with indicators. Very few health facilities had no annual plan and clear indicators $38(9.3 \%)$ and $47(11.5 \%)$ of respondents replied they had no idea about it. Means of communication with the higher offices such as sub city and Addis Ababa city administrative health bureau was commonly made using reports $236(57.8 \%)$, followed by supervision 208 (50.4\%) and seminars/meeting were $134(32.8 \%) .{ }^{1}$ Further, respondents answered that $208(51 \%)$ of the health institutions received feedback from sub city with time intervals of $67(16.4 \%)$ monthly, 37 (9.1\%), quarterly 34(8.3\%) weekly, 26(6.4\%) every two weeks $26(6.4 \%)$ daily, semiannually and annually $18(4.4 \%)$. The feedback was given by means of meetings on HMIS as $265(65 \%)$ of respondents replied. The performance monitoring team meeting $133(27.7 \%)$ replied that meeting session were made regularly, and $152(37.3 \%)$ replied the meetings were made not regular time intervals. Most of the respondents, 370(90.7\%), were heard about HMIS yet only $303(74.3 \%)$ have got training on HMIS. Among these 152 $(37.2 \%)$ respondents were get training last year, 128(31.4\%) trained before a year and 22(5.4\%) trained two years ago.

Factors associated with the utilization of HMIS

The socio-demographic factors, awareness about HMIS, presence of HMIS unit and focal person, burden of duties on HMIS health workers, the practice of using generated information, feedback, availability of computers, and presence of technical supports and attitude of workers towards HMIS were taken as factors that would affect the utilization of HMIS. P-values less than 0.2 were selected and used to multiple logistic regressions (Table 5)

Table 5. Logisticregression of utilization of HMISwith predictor variables in the Addis Ababahealth centers July 2011.

\begin{tabular}{llllll}
\hline Variables & Yes & No & COR $(\mathbf{9 5 \%}$ CI) & $\begin{array}{l}\text { Adjusted OR } \\
\mathbf{9 5 \% C I}\end{array}$ & P. Value \\
\hline Service Year & & & & & \\
$1-5$ & 108 & 167 & $0.28(0.86,0.95)$ & $0.33(0.85,1.32)$ & 0.11 \\
$6-10$ & 32 & 43 & $0.33(0.93,1.17)$ & $0.36(0.08,1.56)$ & 0.17 \\
$11-15$ & 14 & 15 & $0.41(0.10,1.65)$ & $0.53(0.10,2.61)$ & 0.43 \\
$16-20$ & 7 & 9 & $0.34(0.74,1.60)$ & $0.34(0.06,1.90)$ & 0.22 \\
$20+$ & 9 & 4 & 1 & & \\
Salary & & & & & \\
$<1249$ & 45 & 55 & $1.42(0.86,2.37)$ & $1.09(0.60,1.97)$ & 0.76 \\
$1250-2249$ & 66 & 80 & $1.44(0.91,2.27)$ & $1.19(0.68,2.06)$ & 0.53 \\
\hline
\end{tabular}

1 The sum exceeds the total number of respondent because of multiple responses

\begin{tabular}{|c|c|c|c|c|c|}
\hline Variables & Yes & No & COR $(95 \% C I)$ & $\begin{array}{l}\text { Adjusted OR } \\
95 \% \text { CI }\end{array}$ & P. Value \\
\hline$>2250$ & 59 & 103 & 1 & & \\
\hline \multicolumn{6}{|l|}{ Training } \\
\hline Yes & 139 & 164 & $2.02(1.25,3.25)$ & $1.52(0.88,2.62)$ & 0.131 \\
\hline No & 31 & 74 & 1 & & \\
\hline \multicolumn{6}{|c|}{ Personnel specifically to HMIS } \\
\hline Yes & 123 & 151 & $1.50(0.98,2.31)$ & $0.95(0.55,1.62)$ & 0.858 \\
\hline No & 47 & 87 & 1 & & \\
\hline \multicolumn{6}{|c|}{ Other duties too } \\
\hline Yes & 94 & 116 & $1.30(0.87,1.93)$ & $0.89(0.55,1.44)$ & 0.664 \\
\hline No & 76 & 122 & 1 & & \\
\hline \multicolumn{6}{|c|}{ Data converted in to information } \\
\hline Yes & 155 & 150 & $6.06(3.35,10.95)$ & $3.44(1.82,6.51)^{*}$ & 0.001 \\
\hline No & 15 & 88 & 1 & & \\
\hline \multicolumn{6}{|c|}{ Received feedback } \\
\hline Yes & 109 & 99 & $2.50(1.67,3.76)$ & $1.48(0.92,2.37)$ & 0.103 \\
\hline No & 61 & 139 & 1 & & \\
\hline \multicolumn{6}{|c|}{ Use generated report at department level } \\
\hline Yes & 142 & 127 & $4.43(2.74,7.15)$ & $2.78(1.65,4.68)^{*}$ & 0.0001 \\
\hline No & 28 & 111 & 1 & & \\
\hline \multicolumn{6}{|c|}{ Annual plan } \\
\hline Yes & 147 & 176 & $2.25(1.33,3.81)$ & $1.50(0.85,2.75)$ & 0.187 \\
\hline No & 23 & 62 & 1 & & \\
\hline \multicolumn{6}{|c|}{ Presence of computer } \\
\hline Yes & 129 & 127 & $2.75(1.78,4.24)$ & $2.08(1.29,3.35)^{*}$ & 0.003 \\
\hline No & 41 & 111 & 1 & & \\
\hline \multicolumn{6}{|c|}{ Technical Support } \\
\hline Yes & 100 & 100 & $1.97(1.32,2.93)$ & $1.69(1.09,2.63)^{*}$ & 0.019 \\
\hline NO & 70 & 138 & 1 & & \\
\hline \multicolumn{6}{|c|}{ Attitude on HMIS } \\
\hline Positive & 127 & 148 & $1.79(1.16,2.77)$ & $1.10(0.65,1.86)$ & 0.698 \\
\hline Negative & 43 & 90 & 1 & & \\
\hline
\end{tabular}

variables that are significant at $\mathrm{p}<0.05$ level

Utilization of HMIS was compared with sociodemographic variables, and other key variables like training, conversion of data into information, staff attitude on current HMIS, HMIS focal person, burden of duties, feedback, use generated report at department level, preparation of annual plan, and availability of computer and computer programs were analyzed. Salary, level of education, year of services, HMIS person, burden of duties, and training were significantly affected utilization of HMIS before adjusting confounders.

In multiple logistic regressions analysis results showed that rate of data conversion into information, $[\mathrm{AOR}=3.44$, 95\% CI: 1.82, 6.51], converted the collected data in to information had 3.44 times more likely to utilize HMIS as compare to those unit/department didn't converted the collected data in to information. use of generated report at department level [AOR $=2.78,95 \%$ CI: 1.65, 4.68], those who use the generated report at department level had 2.78 times more likely to utilize HMIS as compare to those didn't use generated report. availability of computer in the institution [AOR $=2.08,95 \% \mathrm{CI}: 1.29,3.35$ ], the institution have computer for data base had 2.08 times more likely to utilize HMIS as compare to those didn't use computer for data base and provision of technical support [AOR $=1.69$, 95\% CI: $1.09,2.63]$ those who get technical support from concerned bodies about HMIS issues had 1.69 times more likely to utilize HMIS as compare to those who didn't get 
technical support were significantly enhanced utilization of HMIS. However, majority of selected factors were not statistically significant in multiple logistic regressions.

Qualitative results

In this qualitative study the results found from observation check list format was completed in all studied health facilities and analyzed using thematic approaches. The figure below illustrates the general overview of the main and sub thematic findings. Figur-6

Infrastructure
In all studied health facilities observational study was focused on data management unit, Medical Record Unit (MRU)/card room and HMIS units of the study health centers. It is very difficult to measure the exact room size of card rooms (MRU). Most of the observed health facilities had spacious card rooms with standard size with sufficient aeration and moving spaces. All observed health centers had four and three windows that provided service of fast tracking system (figure 6)

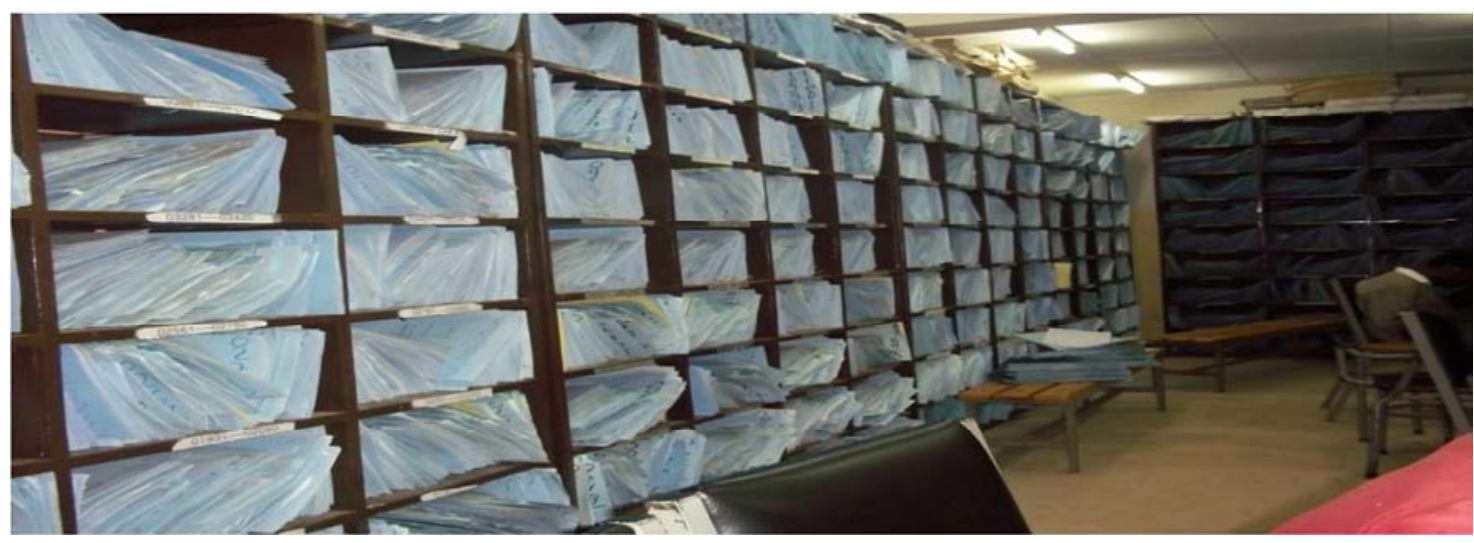

Figure 6. Standard MRU with \& sufficient aeration and moving spaces A. A health center 2011.

Some of health centers had small and staffed with materials with little aeration and open spaces. Even if some of them had standard card room size had only one and two windows for the fast tracking system purpose and they had staffedand suffocated card rooms (Fig 7).

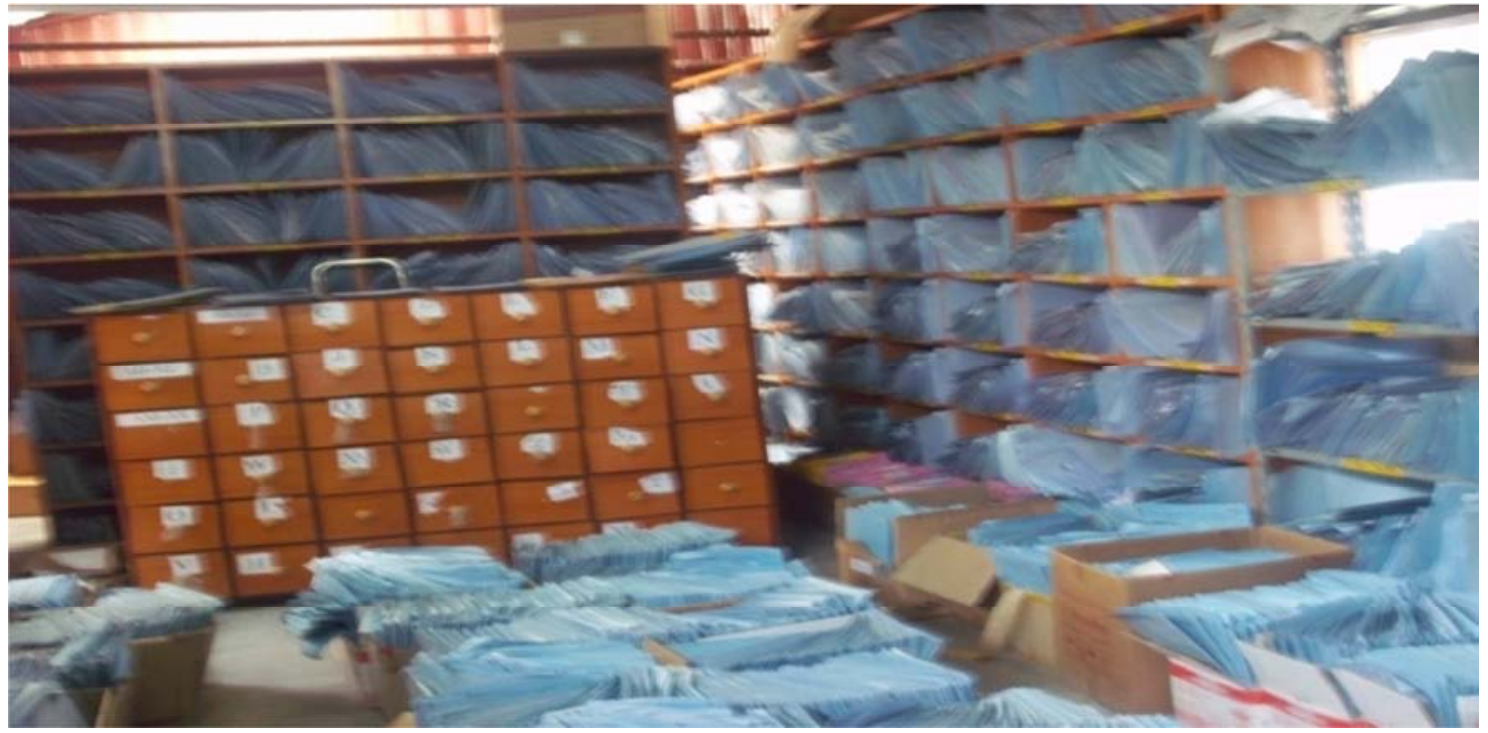

Figure 7. Medical record units with small size and staffed by materials with little aeration and open space, July 2011.

Equipments and materials in the HMIS units

All of the health centers had computer but the number of computers vary. For instance, majority health center had minimum one computer and maximum two to four. All health centers mostly used the computers for storing and analyzing their datasets. Among the studied health facilities $16(61.5 \%)$ of them had HMIS unit while the remaining 10(38.5\%) health centers didn't have HMIS unit but they had HMIS focal persons and furnished card rooms. Only five of the health facilities had internet and other computer networking system.

All health centers have HMIS focal person, of these three health centers had $2 \& 3$ personnel's, but the rest health facilities had single personnel with the qualification of IT 12 (46.2\%), nurse $11(42.3 \%)$, statistician and secretary $3(9.3 \%)$. Standard shelves and MPI box are important equipments in card rooms to store client health information. All of them had a certain number of shelves although they did not satisfy. 
Regarding standard MPI box all of them had only one standard MPI box.
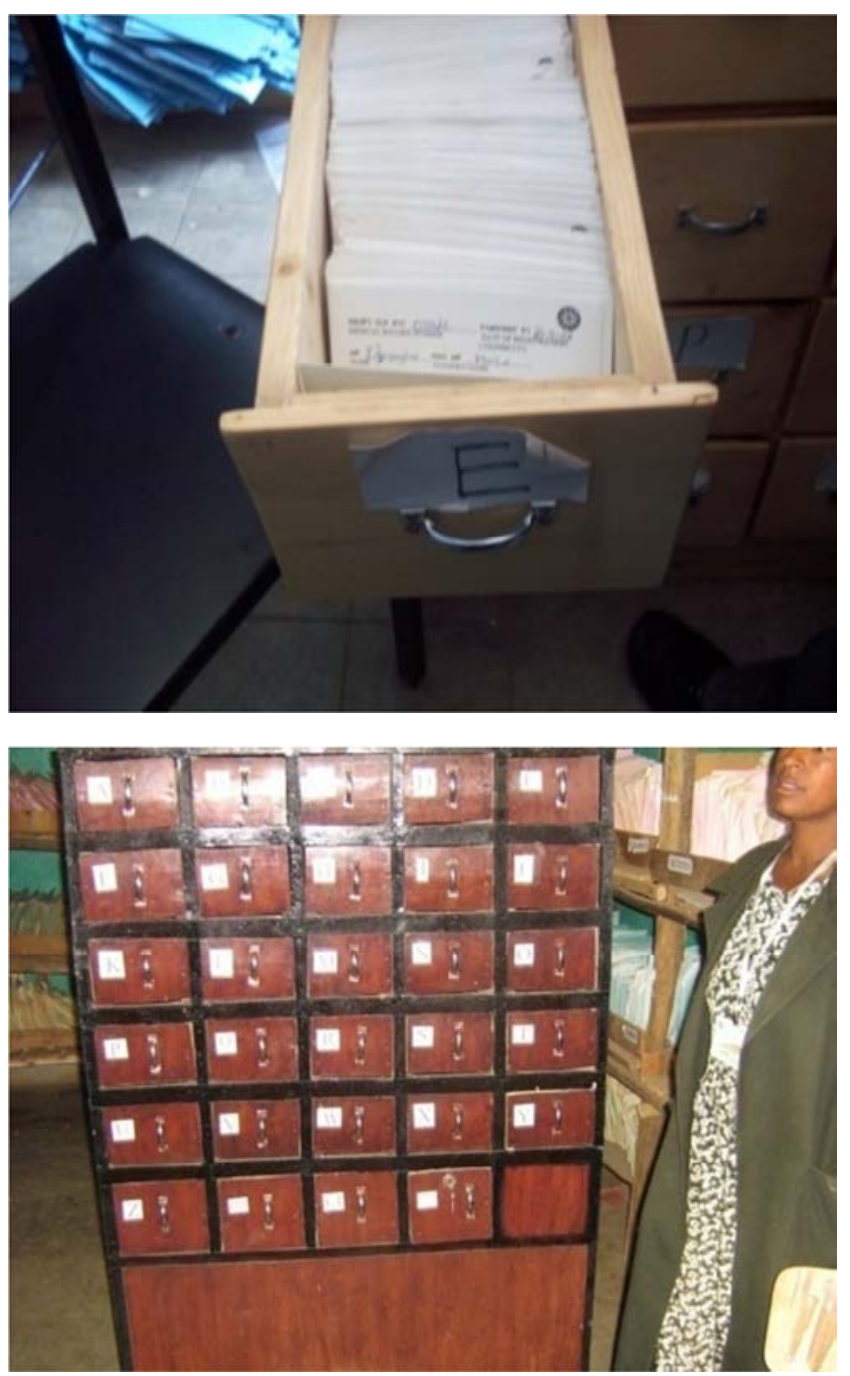

Figure 8. Health center MPI box July 2011.

\section{HMIS Use}

The HMIS unit focal persons had taken training in HMIS in almost all of the health facilities. However, all HMIS units had no special allocation of budget for they used budget of printing and publishing to undertake the activities of HMIS. The training of HMIS has been given by foreign aid organizations particularly from Tulane University. Although the funding and training of this aid organization will phase out, the health facilities directors believed to pursue the activities of the HMIS unit by their own budget and plans in the future. In the health facilities perhaps in all of them there is performance monitoring team that comprised at least 4 members and at most 12 members. Each team includes the medical director, HMIS focal person and each department head the performance monitoring team has log books and wrote a minute during their meeting. The performance monitoring team meeting was about 4 to 5 times in a year.

\section{Discussion}

The data generation mechanisms were predominantly based on tally sheet, registers reports, their combination $199(48.8 \%)$. This result indicated that a great deal of data can be generated in various formats and systems that would increase the quality of data generated. Western pacific developing health management information a practical guide for developing countries and SINAIS Assessmentthe accuracy of data quality also measured by the number of data registered reported and documented increase the data quality $[29,30]$. This research result have a certain association with this study result that showed use of several formats to generate health data believed to increase data quality.

In this study, the general utilization on health management information rate was found to be $41.1 \%$. Even though most of the developing countries have low utilization rate, finding of utilization of information in Addis Ababa health centers was slightly higher as compared to the results of a study done by Gashaw [23] in North Gonder 2006 which, the utilization rate of $22.5 \%$ in all the study units and 8\% in HIV/AIDS units. Moreover, Campbell in Ghana and Maria G. N. Mosoke et al. in Uganda as identified $10 \%$ and $20 \%$ of the health system utilize the health information for decision making and evaluating and controlling [31,32]. In contrast, our study showed slight increase in comparison of these studies perhaps it may be due to training and supportive supervision of Addis Ababa Health Bureau and NGOs such as Tulane University. Utilization of information in this study area predominantly to the sake of generatedreport were $269(65.9 \%)$ this result compared to the result reported in India Study of Data Utilization in the Health Sector was for the sake of planning this deference may be due to infancy stage of utilization of information at health center in A. A [33].

This study also tried to see factors that might affects the utilization of HMIS that unit/department level and the rate of conversion the collected data in to information it has been showed that health centers had 3.44 times more likely to utilize HMIS as compare to those unit/department didn't converted the collected data in to information. Other factor which may affects the utilization of HMIS those who use the generated report at department level had 2.78 times more likely to utilize HMIS as compare to those didn't use generated report at department level, the institution have computer for data base had 2.08 times more likely to utilize HMIS as compare to those didn't use computer for data base and those who get technical support from concerned bodies about HMIS issues had 1.69 times more likely to utilize HMIS as compare to those who didn't get technical support. A similar result obtained in a study in Korea indicated that the availability of personal computers and connection of computers with administrative offices and departments increase the average time for inquiry and record that ultimately developed health management information system in the health centers [34]. 
Planning budget is an indication of systematized and programmed functioning of a certain sector of the institution. In this study the 26 health centers did not have budget to run HMIS this result was similar to a result obtained through observation. The works of HMIS was done from the finance of printing and publishing. This indicated that the system was not organized well. In the same relation, the study on design and implementation of HMIS in Malaw indicated that there is very little improvement in the design and planning of information system that hampers the implementation of HMIS in the health facilities [35].

In the same token study report by the National Health Information System Assessment Report (NHISAR) the HMIS of Ethiopian health centers indicated that there is lacks adequate resources to effectively maintain and upgrade the status of health information system to a level that meets the health information requirements of the country [7]. The above mentioned studies diametrically strengthen and associated with the result of this research.

The interaction between the health facilities and higher health offices would have effect on the performance and strength of HMIS in the health centers. The results of this research showed that there are promising relationship and feedback between the health facilities and higher health offices and among the department of the health center. Reports were prepared and send to higher management offices in monthly basis. The presence of reports in the past 90 days indicated that the HMIS is functional at this level. Furthermore, from this study it was observed that 51 percent of respondents answered that they were received feedback from HMIS departments. However, 28.4 percent of them did not have idea whether there would be feed back or not. This is a significant figure that strongly affects the relationship and feedback horizontally and vertically. Together with this the frequency of interaction between the offices was mainly monthly using reports and sometimes visits and seminars.

The uses of information within the department were encouraging it was about 63.9 percent of the respondents answered it was used by the unit and the health institution. The information primarily used to the benefit of the user/clients and for planning and decision making. In general, the results showed that there is strong organization structure in operation but weak or poor form of organizational structure with work and financial sources.

The results of the study indicated that the HMIS performance showed development in comparison with the previous studies in Addis Ababa [16]. However, in comparison with other East and South African countries HMIS utilization and implementation the result showed poor organizational and rational decision using information. For instance, in Malawi there was good design of information system and encouraging feedback system [35]. In Kyrgyzstan the implement of HMIS bytraining workers with skills to monitor and plan their health information system improved the quality of data [36].

Majority of health centers have performance monitoring team $265(65 \%)$ this team is established in health facilities to maximize information use for decision making at the World Health Organization specifically institution level. However, in this study all $\mathrm{H}$. C had performance monitoring team three to four times per year meeting except one H. C. In contrast, the descriptive result showed that $113(27.7 \%)$ meet regularlybutobservation log book result meeting not regularly 3 to 4 times per year meeting this result show the difference between descriptive result and observation result. FMOH report frequency of meeting is monthly. This indicated that there is no strong and smooth relationship.

In all health centers studied, there is HMIS unit, responsible for data generation only $61.5 \%$ of the study units have separate HMIS units, but $38.5 \%$ of the respondent agreed that they did not have separate HMIS unit to collect and process the data. All studied health centers had HMIS focal person. The qualification of HMIS focal persons include in the study health facilities includes IT, nurses, and statisticians. Among these professional $46.2 \%$ are IT professionals, $42.3 \%$ nurses and the rest $9.3 \%$ statistician and secretary. Besides, the BPR report on Addis Ababa showed the highest levels of staffed HMIS units which account $95 \%$ of the public health $\mathrm{H}$. C. This report had very similar figures with respect to staff of HMIS in health facilities indicated in this study (16). However, the presence a single person in HMIS would cause the failure of HMIS function when the individual absent from work for various reasons. This problem is also identified by the national health information report of FMOH [7]. This similar of the study maybe due to reformed HMIS. The IT equipment such as computer, printer, fax, and internet are believed to be an essential infrastructure required for the development in HMIS as they have great importance in helping to improve data storage and economize time and improve accuracy to support the enhancement of health information system [6]. However, in the study it was found that health centers were not fully equipped with the essential infrastructures. Among the 26 health centers all have a minimum one computer and 5 health centers have networked computerized system. Concerning other electronic facilities such as printer and fax machine were not observed in all the study areas.

All HMIS focal person are trained on HMIS, study show that from North Gondar assessment of health information system stated that only $23.8 \%$ of the individual were given training on health information system which is almost all our study area gets training. The difference may be due to supportive NGOs Tulane University given training all health workers.

In the study it has been obtained that $81 \%$ health professional record their daily activities and the services provided and $73.8 \%$ of the study units take data collection as part of their duty but according to Assessment of health management information system in Addis Ababa more than $80 \%$ out of the total service are registered by health professionals [16]. However, the remaining is registered by other personnel and also $78 \%$ of the respondents have stated that they take data registration as part of their duty. This 
result is similar to our finding.

Most of the health facilities had annual plan with clear indicators. They collected the HMIS data using computers and paper formats designed in accord with the services. However, they hardly had guidelines and procedural manuals of HMIS which indicated that they have little knowledge about the HMIS. Concerning data processing and analyzing there was the initiation but it was not developed. 62.7 percent of the respondents answered they fed the data on computers but the software was incomplete to analyze the information. The researcher learnt that in some health facilities SMART software was provided by some NGOs but it was commonly incomplete and software not used friendly. Other study report by the SINAIS Assessment Report information technology and HIS software are user-friendly this deference may be due to lack of computer knowledgeand technology in our situation [36].

In East Africa the implementation of HMIS faced challenge in general, this basically stemmed from the poor adoption of innovated HMIS systems, poor equipments and trained manpower and little commitment for the duty [37] Ethiopia is not exception, some projects from Europe were come to Addis Ababa to introduce systems to institutionalize and customize the information system but failed to fulfill and innovate the health management information system due to several factors [6].

In related study. Gladwin $\mathrm{J}$ et al. Rejection of an innovation health information management [38] it has been indicated that there is strong challenge in East African to diffuse organizational structure and adoption of various tools that help to implement health management information system.

Demand for HMIS, confidence, motivation and competence of personnel affect HMIS processes and performance directly hence how an individual feels about the utility, his/her skill and confidence in performing that task all affect the likelihood of that task being performed [39]. The researcher noted that most of the medical record unit staff is working under inconvenient situation with poor office facilities and inconvenient space, limited access to information technologies and absence of staff benefit like intensive training opportunities which could hamper staff motivate and ultimately affecting HMIS. Absence of regular monitoring and feedback mechanism is another factor limiting the motivation and confidence of data management staff to aspire for quality outputs.

The result of the question whether the workers in the health institution current attitude toward HMIS are indicated that $67.4 \%$ of the respondents answered the workers had positive attitude toward the duty of collecting data. The strong commitment was observed in collecting and storing the health information with poor motivation working environment. This support strongly the implication of HMIS is accepted positively by the health workers. The problem is emanating from the organization structure and furnishing of the system with equipment and budget allocation are the identified curtails in the utilization of HMIS in this study.
Similarly, study conducted in Uganda [40]showed that organizational issues were not pleasing in the implementation of HMIS. The results documented that diffusion of innovation in organizational structures were resisted and become common barrier to establishing and sustaining effective routine HMIS. Similarly, a study in Zambia [41]showed that the existence of different forms of information in the organizational environment, including those provided by the conventional HMIS, affected the implementation of effective HMIS.

\section{Conclusions}

The implementation of HMIS in Addis Ababa health centers was initiated but hardly advanced. In general, the utilization of health management information system at health centers was slightly higher as compared to previous study but in general still low.

Most of the data and information was generated at the health center from routine day to day service based records of the patients who visit the health center. Register book, tally sheet and forms are the most common data collection tools currently used this indicates improve data quality.

Among many factors affecting the utilization of HMIS only data converted in to information, use of generated data at department level, health centers have computer and HMIS get technical support was found to be significantly association.

\section{Recommendations}

To public health centers in A. A

1. Feedback and supervisory support to enhance staff motivation and commitment.

2. Performance team evaluation was encouraging but this team should have regular schedule that strongly lined with the plan of HMIS.

3. Data has to be used at institutional level for decision making not just for the sake of forwarding to the upper level in the form of report.

4. Standardized and user friendly data collection, analysis and reporting format should be in place.

To Addis Ababa City Administrative Health Bureau and Sub cities:

1. Management has to assign adequate human resource for data management unit and should be equipped with basic ICT infrastructures.

2. HMIS unit should have their own budget and strategy like any other health facility units.

3. Renovation on the HMIS organizational structure.

4. Intensive and continuous training for the workers should be given.

5. There has to be periodic and regular feedback system to the HMIS unit from the higher management bodies.

6. Strategic guidelines should be developed that help to govern the overall process. 


\section{References}

[1] Angelo S Nyamtema. Bridging the gaps in the Health Management Information System in the context of a changinghealth sector. Medical Informatics and Decision Making. 2010.

[2] Theo Lippeveld, Rainer Sauerborn, Claude Bodart. Design and implementation of health information systems. World Health Organization Geneva. 2000; 11-45.

[3] World Health Organzation. Health Information System Development and Strengthening Gaidanceon need assessment for national health information system development. 2000 January; 6-11.

[4] HMIS Business Process Re-engineering Assessment Report HMIS Core Process. HMIS Reform Team. 2006 Sep.

[5] FMOH, Assessment of the Ethiopian National Health Information System. Addis Ababa. 2007.

[6] Anagaw Shegaw. Special Issues on IT and Health Care in Developing Countries, Department of Informatics., University of Oslo, Norway. 2010; Vol. 4 No. 11-17.

[7] Honest C. Kimaroa and José L, Nhampossab. The challenges of sustainability of health information systems in developing countries: comparative. case studies of Mozambique and Tanzania. 2007.

[8] Ayano M. Assessment of Health Managment Information System at Public Hospital in Addis Ababa. A. A. U Thesis research. 2009.

[9] Health Care Review. Vol. 25 (2): 74-78.

[10] Potomac. Issues and Innovation in Routine Health Information in Developing Countries. Keynote address at the Workshop. 2000 March; 14-6.

[11] Stefano Calciolari. Information integration in health care organizations the case of a European health system. 2010 Sep.

[12] Rod Ward et al. The Attitudes of health care staff to information technology: a comprehensive review of the research literature Health Information and Libraries. 2008; Vol. 25: 81-97.

[13] Joost Hoppenbrouwer \& Christine Wamunyima Kanyengo. Current access to health information in Zambia. Health information and Libraries. 2007; Vol. 24: 246256.

[14] Arthur Heywood, University of Western Cape/HISP Jon Rohde, MSH/EQUITY Project. Using Information for Action. University of Western Cape School of Public Health.

[15] Planning and Programming Department, Ministry of Health of Ethiopia. Health Metrics Network Ethiopia Health Information System: Review and Assessment Version 4.00. 2007 May.

[16] Alganesh. Assessment of Health Managment Information System in Addis Ababa Health Bureau. 2005 Jun.

[17] Sameh El-Saharty, Sosena Kebede, Petros Olango, Banafsheh Siadat. Ethiopia Improving Health Service Delivery. 2009 Aug.

[18] Federal Ministry of Health. Health Management Information
System (HMIS) /Monitoring and Evaluation (M\&E) Information Use Guidelines and Display Tools HMIS / M\&E Technical Standards: Area 4 HMIS Reform Team. 2008 Jan.

[19] HMIS Reform Team. Federal Ministry of Health HMIS / M\&E Information Use Guidelines and Display Tools. 2007 May.

[20] Hedberg Calle,. Health Information System Progress with Caveats, an integration HMIS / M\&E Redesign: Technical Standards. 2003.

[21] WHO. Priority Intervention for Strengthening National Health Information System. 2004.

[22] Lungo Juma Hemed. Data Flows in Health Information Systems, University of Oslo, Department of Informatics, Norway. 2003 May.

[23] Andargie G. Assessment of Utilization of Health Information System at District Level with Particular Emphasis to HIV/AIDS Program in North Gondar Zone Amhara National Regional State. A. A. U. 2006 Jun.

[24] Federal Ministry of Health. Health Sector Strategic Pl (HSDPIII) 2005/6-2009/10 Planning and Programming Department. 2005.

[25] Federal Ministry of Health. Health Management Information System (HMIS) /Monitoring and Evaluation (M\&E) Strategic Plan for Ethiopian Health Sector. 2008 Jan.

[26] Addis Ababa City Adminstrativ Health Breua. Standard Operating Procedures for Decentralizing ART Initiation and follow up care to Public Health Centers and Private Facilites. 2006 Oct.

[27] UNFPA. Summary and Statistical Report of the 2007 Population and Housing Census A. A. 2008 Dec.

[28] Worku A. Empowerment of Youth club: Experience, Opportunities \& Challenges. The case of "Sele Tselote Egi" Youth Reproductive Health/HIV/AIDS club in Oromia and "Kal" $\mathrm{RH} / \mathrm{HIV} / \mathrm{AIDS}$ club in Addis Ababa Region-Ethiopia. Addis Ababa University School of Graduate Program. 2006 Jul.

[29] World Health Organzation. Regional office for the western pacific developing health management information apractical guide for developing countres. 2004.

[30] Aquil, A etal. Guanajuato SINAIS Assesment. USAD 2010.

[31] B. Campbell, S. Adjei, A. H. eywood: From data to decision making in health, the evaluation of a health management information system; Royal tropical Institute, Amesterdam the Netherlands, 1996.

[32] Maria G. N. Musoke etal; information and its value to health workers in rura Uganda: a qualitative perspective. Black well science Ltd, health bibaries review,. 2000; 17, pp 194-202.

[33] Scott Moreland, Sutapa Agrawal; Data Utilization in the Health Sector of India: United States Agency for International Development (USAID) 2009.

[34] Chae, Y. M. et al. Implementing Health Management Information Systems: Measuring success in Korea's health centers. Health Management and Planning International Journal 1994; Vol. 9: 341-348.

[35] Chaulagai, C. N. et al. Design and implementation of a health management information system in Malawi: issues, innovations and results. School of Hygiene and Tropical Medicine, London, Oxford University 2005. 
[36] Weeks, R. Mark et al. improving the monitoring of immunization services in kyrgyzastan Health Policy and Planning. 2000; 15 (3): 279-286.

[37] Gladwin, J. et al. Strengthening health information management in an East African country using external training materials. HealthInformatics Journal. 2000; 6: 9-14.

[38] Gladwin J et al. Rejection of an innovation health information management training materials in east Africa, Health Policy and Planning 2002; 17 (4): 354-361.

[39] Aqil, Anwer, Lipeveld, Theo and Dairiku Hozumi. PRISM framework: a paradigm shift for designing, strengthening and evaluating routine health information systems. 2009.

[40] Gladwin J. et al. Implementing a new health management information system in Uganda Health Policy and Planning. 2003; Vol. 18 (2): 214-224.

[41] Mutemwa, R. I. HMIS and decision making in Zambia: rethinking information solutions for district health management in decentralized health systems. Oxford University press in association with London School of Hygiene and Tropical Medicine. 2005; 10. 1093. 